\title{
Painful suprapatellar mass
}

\section{Carol Pui Yang Chien ${ }^{1} \cdot$ Victor Kam Ho Lee ${ }^{2}$ (1)}

Received: 30 August 2021 / Revised: 3 December 2021 / Accepted: 8 December 2021 / Published online: 20 December 2021 (c) ISS 2021

History: A 64-year-old male presented to our orthopaedics department complaining of acute worsening of left anterior knee pain after trauma. Physical examination of the left knee reviewed a tender and mobile soft tissue mass at the suprapatellar region (Figs. 1, 2, 3, and 4).

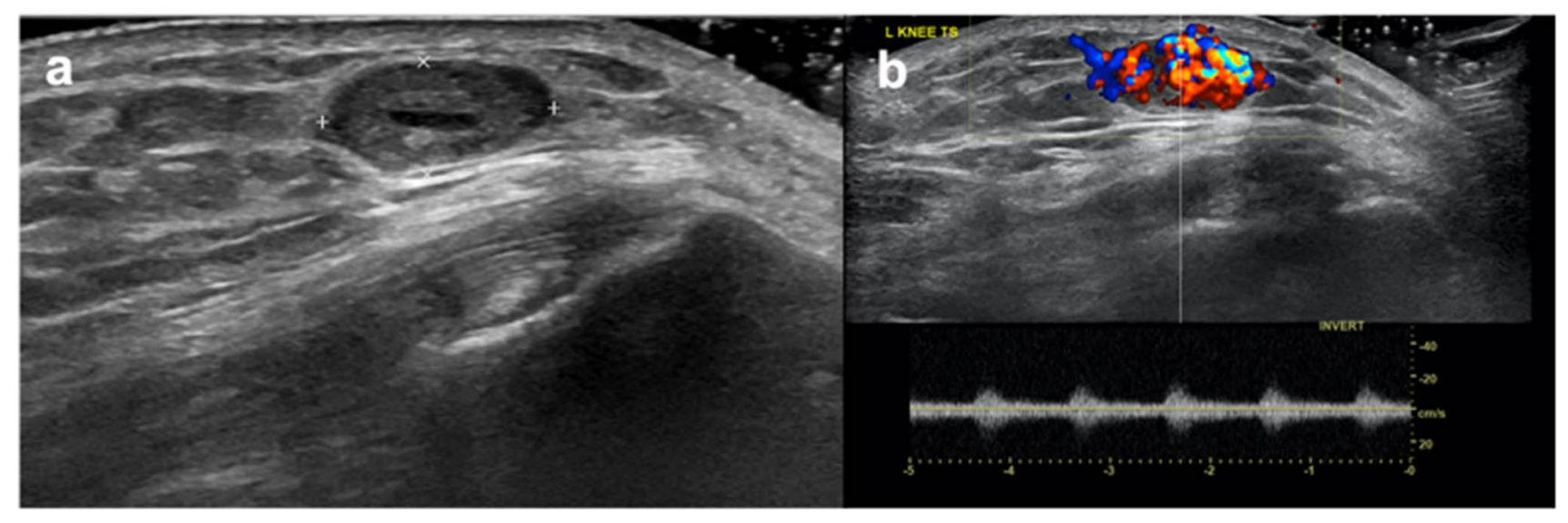

Fig. 1 USG of the left knee. a Grayscale USG image. b Spectral Doppler USG image

The diagnosis can be found at https://doi.org/10.1007/s00256-02103973-8

Victor Kam Ho Lee viclkh88@gmail.com

Carol Pui Yang Chien chienpyc@gmail.com

1 Department of Radiology, Queen Mary Hospital, Pokfulam, Hong Kong, Hong Kong

2 Imaging and Interventional Radiology Centre, CUHK Medical Centre, 9 Chak Cheung Street, Shatin, New Territories, Hong Kong, Hong Kong 


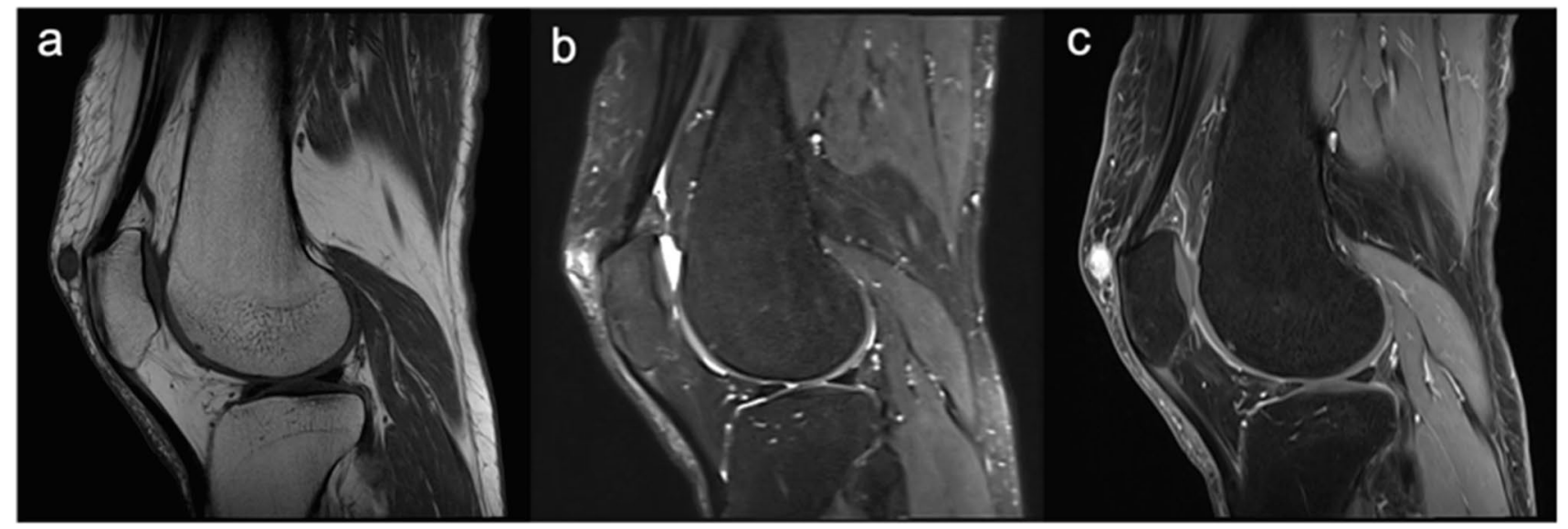

Fig. 2 MRI of the left knee. a Sagittal T1W TSE image. b Sagittal T2W Dixon water image. c Sagittal T1W fat-saturated post-contrast image

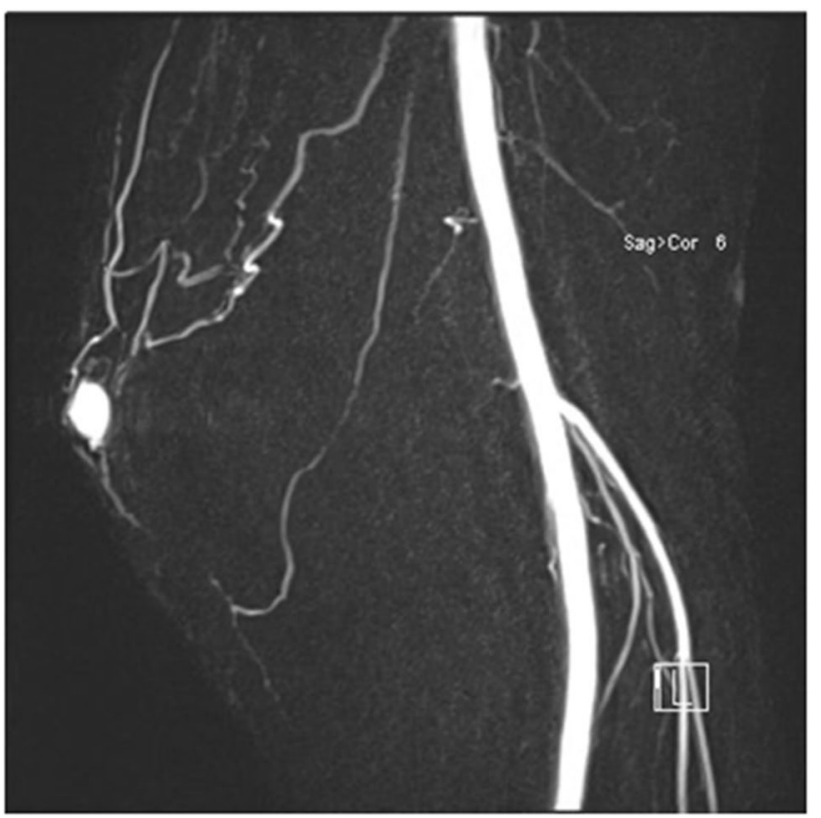

Fig. 3 Full-thickness sagittal maximum-intensity-projection (MIP) image from the time-resolved MR angiography (MRA) 


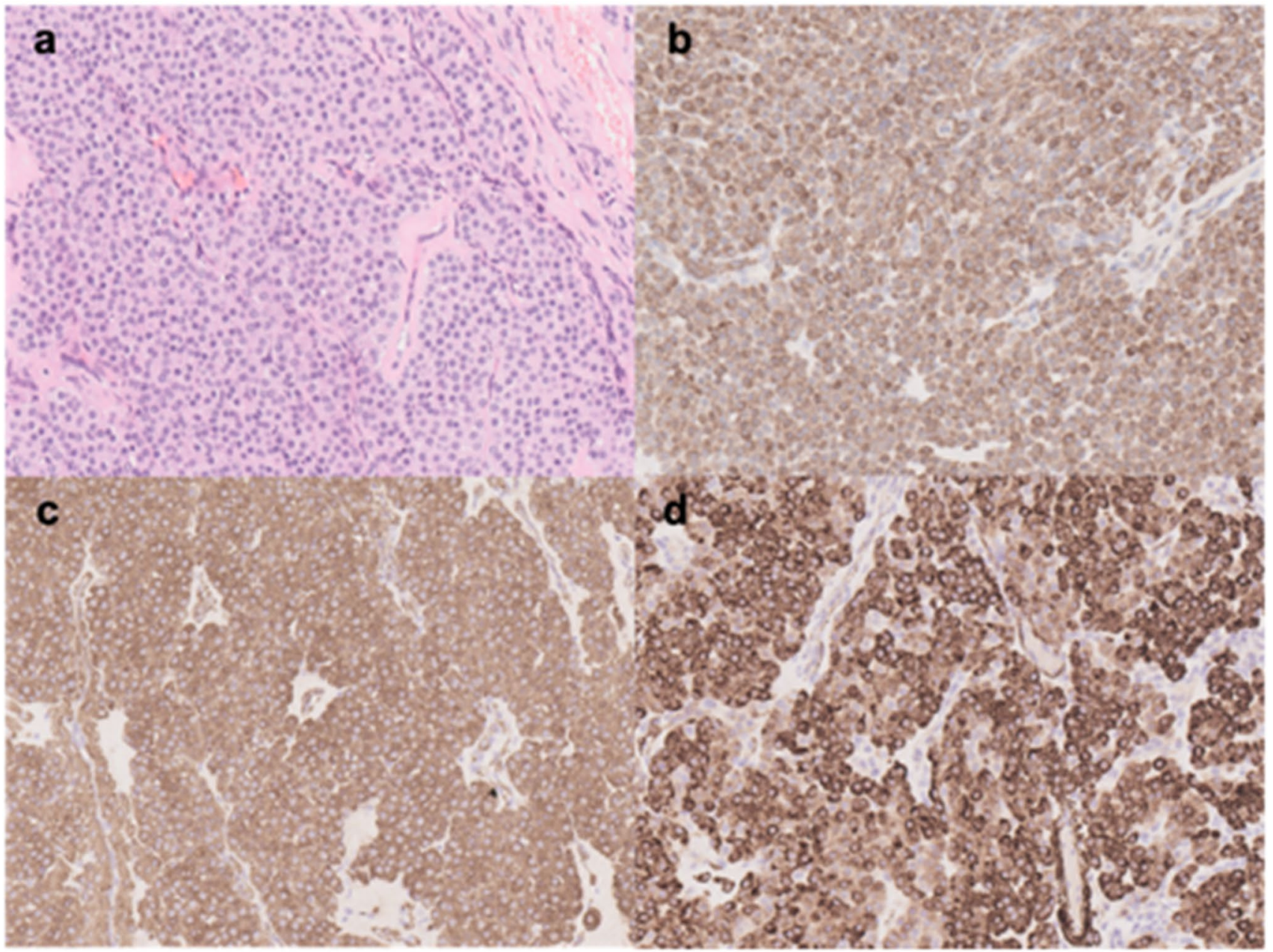

Fig. 4 Histopathology images of the surgical specimen. a Hematoxylin and eosin stain. b Calponin immunostain. $\mathbf{c}$ Actin immunostain. d h-Caldesmon immunostain

\section{Declarations}

Conflict of interest The authors declare no competing interests.

Publisher's Note Springer Nature remains neutral with regard to jurisdictional claims in published maps and institutional affiliations. 